\title{
Oridonin enhances the anti-tumor activity of gemcitabine towards pancreatic cancer by stimulating Bax- and Smac-dependent apoptosis
}

\author{
Dian-Lei Liu ${ }^{1,2 \#}$, He-Qi Bu ${ }^{3 \#}$, Wen-Long Wang ${ }^{2}$, Hua Luo ${ }^{2}$, Bo-Ning Cheng ${ }^{2}$ \\ ${ }^{1}$ Department of General Surgery, the Womens Hospital, School of Medicine, Zhejiang University, Hangzhou, China; ${ }^{2}$ Department of General \\ Medicine, Guangxing Affiliated Hospital of Zhejiang Chinese Medical University, Hangzhou, China; ${ }^{3}$ Department of Coloproctology Surgery, \\ Tongde Hospital of Zhejiang Province, Hangzhou, China \\ Contributions: (I) Conception and design: All authors; (II) Administrative support: B Chen; (III) Provision of study materials or patients: D Liu, H Bu; \\ (IV) Collection and assembly of data: W Wang, H Luo; (V) Data analysis and interpretation: D Liu, H Bu; (VI) Manuscript writing: All authors; (VII) \\ Final approval of manuscript: All authors. \\ \#These authors contributed equally to this work. \\ Correspondence to: Bo-Ning Cheng. Guangxing Affiliated Hospital of Zhejiang Chinese Medical University, No.453 Tiyuchang Street, West Lake \\ District, Hangzhou, China. Email: boning2006@126.com.
}

Background: Oridonin has been shown to exhibit potent anti-tumor activity, but the exact mechanisms underlying this activity remains unclear. Here, we investigated whether oridonin could synergistically enhance the activity of gemcitabine against BxPC-3 pancreatic cancer cells.

Methods: CCK-8 assays were conducted to determine cell viability. The cellular morphology was observed under light microscope and compared with normal cell. Apoptotic cells were quantified by flow cytometry. RT-PCR, Western blot analysis and immunohistochemical methods were employed to analyze related-gene and protein expression. A xenograft tumor model was conducted whereby BxPC-3 cells were introduced into nude mice to detect anti-tumor effects in vivo.

Results: In vitro, oridonin inhibited the proliferation of BxPC-3 and Panc-1 cells cells in a concentration and time dependent manner. In addition, oridonin dose-dependently induced Panc-1 cellular morphology changes. Besides, In BxPC-3 cells oridonin potentiated gemcitabine-induced apoptosis. oridonin induced Bax translocation from cytosolic to mitochondrial compartments. This was accompanied by the release of the apoptogenic protein Smac and inhibition of its downstream target XIAP. These effects were further enhanced by combined treatment with oridonin and gemcitabine. In vivo, both oridonin alone and in combination with gemcitabine significantly suppressed tumor growth in a Bax- and Smac-dependent manner.

Conclusions: Oridonin can potentiate the effects of gemcitabine through Bax- and Smac-dependent mitochondrial signaling pathways in BxPC-3 pancreatic cancer cells. Therefore, oridonin has the potential to be used clinically in the treatment of pancreatic cancer.

Keywords: Oridonin; gemcitabine; Bax; Smac; pancreatic cancer

Submitted Dec 28, 2019. Accepted for publication Jun 11, 2020.

doi: $10.21037 /$ tcr-19-3000

View this article at: http://dx.doi.org/10.21037/tcr-19-3000 


\section{Introduction}

Pancreatic cancer is a malignant neoplasm that causes the death of more than 43,000 people per year in the United States (1). Relative to other tumors, it has an extremely poor prognosis and doesn't respond well to conventional chemotherapy. The 5 -year relative survival rate of patients with pancreatic cancer is approximately $6 \%$, with a median survival time after diagnosis of 6 months $(2,3)$. Although surgical resection is the most effective treatment, only $20 \%$ of tumors are surgically resectable (4). Alternatively, a high proportion of patients with pancreatic cancer are required to undergo either radiation therapy or chemotherapy, rather than surgery. Gemcitabine is the standard treatment for pancreatic cancer, but its effectiveness as a monotherapy has been shown to be limited (5). Although tumors may initially respond to gemcitabine treatment, a subset of tumor cells emerge that escape treatment and display acquired resistance. Several innovative trials have been designed to increase treatment efficacy by combining gemcitabine with other chemotherapeutic drugs. Unfortunately, many of these trials were associated with toxicity or lacked significant improvement when compared to gemcitabine alone $(6,7)$. Therefore, the identification of compounds capable of synergizing with gemcitabine and enhancing its efficacy is urgently needed.

Oridonin is a diterpenoid isolated from Rabdosia rubescens and has been reported to have anti-inflammatory, antibacterial, and anti-tumor effects (8). With regard to its anti-tumor activity, previous reports have demonstrated that oridonin induces apoptosis in breast, gallbladder, gastric, liver, and pancreatic cancer cells (9-13). Apoptosis occurs through two principal pathways: the death receptormediated pathway and the mitochondria-mediated pathway (14). Stimulation of mitochondria-mediated pathway results in increased permeability of the mitochondrial membrane and the release of the pro-apoptotic proteins cytochrome c, Smac (second mitochondria-derived activator of caspases, also known as DIABLO), and apoptosis-inducing factor (AIF) into the cytoplasm (15). Our previous study demonstrated that oridonin could augment the cytotoxic effects of gemcitabine against pancreatic carcinoma cells in vitro by downregulating Bcl-2 expression and upregulating Bax expression, resulting in the reduction of the Bcl2/Bax ratio. This facilitated apoptosis via the release of cytochrome-c from the mitochondria into the cytoplasm and subsequent caspase-3 and -9 activation (16). Results showed that oridonin alone, or in combination with gemcitabine, could up-regulate the expression of Smac in BxPC-3 cells.
However, it is currently unknown whether Smac, or Smacregulated gene products, are involved in mediating the proapoptotic effects of oridonin against pancreatic cancer cells.

Therefore, we investigated the effects of oridonin alone, or in combination with gemcitabine, on the growth and apoptosis of pancreatic cancer cells both in vitro and in a mouse xenograft model. Our results demonstrated that oridonin could significantly enhance the anti-tumor effects of gemcitabine against pancreatic $\mathrm{BxPC}-3$ cells both in vitro and in vivo, via mitochondria-mediated apoptosis pathway involving Bax and Smac.

We present the following article in accordance with the ARRIVE reporting checklist (available at http://dx.doi. org/10.21037/tcr-19-3000).

\section{Methods}

\section{Reagents}

Oridonin was obtained from the Beijing Institute of Biological Products (Beijing, China). The purity of oridonin was measured by HPLC and determined to be $99.4 \%$. Oridonin was dissolved in dimethyl sulfoxide (DMSO) to make a stock solution at $10 \mathrm{mmol} / \mathrm{L}$ concentration and stored at $-20{ }^{\circ} \mathrm{C}$. The DMSO concentration was kept below $0.1 \%$ in all cell culture experiments and did not exert any detectable effects on cell growth or cell death. Gemcitabine (Gemzar; Eli Lilly and Company) was stored at $4{ }^{\circ} \mathrm{C}$ and dissolved in sterile PBS at $0.2 \mathrm{mmol} / \mathrm{L}$ concentration on the day of use. The Cell Counting Kit-8 (CCK-8) was obtained from Abcam. Annexin V-FITC/PI apoptosis detection kit was purchased from Biological Development Co. Ltd. The RNA extraction kit was purchased from Life Technologies. cDNA first-strand synthesis kit was obtained from Fermentas. The $2 \times$ Taq PCR MasterMix was purchased from TIANGEN and the mitochondrial/ cytosol fractionation kit was purchased from BioVision, CA, USA. Fetal bovine serum (FBS), trypsin containing EDTA, Roswell Park Memorial Institute-1640 (RPMI1640), Ribonuclease A (RNase A), propidium iodide (PI), DMSO, antibodies against Bax, Smac, X-linked inhibitor of apoptosis protein (XIAP), caspase-9, cleaved caspase-3, $\beta$-actin, and horseradish peroxidase (HRP)-conjugated secondary antibodies (goat-anti-rabbit and goat-anti-mouse) were all purchased from Sigma.

\section{Cell lines and tissue culture}

The pancreatic cancer cell line BxPC-3 and PANC-1 were 
obtained from the American Type Culture Collection (ATCC). All cell lines were cultured in RPMI-1640 supplemented with $10 \% \mathrm{FBS}, 100$ units/mL penicillin, and $100 \mu \mathrm{g} / \mathrm{mL}$ streptomycin. Cells were maintained at $37^{\circ} \mathrm{C}$ in a humidified atmosphere of $5 \% \mathrm{CO}_{2}$. The medium was changed every 48-72 h. Cells were sub-cultured when confluency reached $70-80 \%$, using $0.25 \%$ trypsin at $37^{\circ} \mathrm{C}$.

\section{Cell viability assay}

Cell survival was determined using the CCK-8 kit. Briefly, cells in $\log$ phase were plated in 96-well culture plates $\left(6 \times 10^{3}\right.$ cells per well). After a $24 \mathrm{~h}$ incubation, the cells were treated with the vehicle alone $(0.1 \%$ DMSO) or varying concentrations $(5,10,20,40$, and $80 \mu \mathrm{M})$ of oridonin, followed by 24,48 , and $72 \mathrm{~h}$ of cell culture, or treated with oridonin $(40 \mu \mathrm{M})$ and gemcitabine $(20 \mu \mathrm{M})$ both alone and in combination for $48 \mathrm{~h}$. CCK-8 $(100 \mu \mathrm{L})$ was added to each well for $1 \mathrm{~h}$ before absorbance at $450 \mathrm{~nm}$ was read using a Bio-Tek ELX800 plate reader. Experiments were repeated three times with six replicates per treatment in each experiment. Percent inhibition of cell viability was calculated using the following formula:

Relative \% inhibition $=1-($ dosing absorbance - blank absorbance) $/($ control absorbance - blank absorbance $) \times 100 \%$.

\section{Flow cytometry analysis of apoptosis}

To determine whether oridonin could potentiate the apoptotic effects of gemcitabine in pancreatic cancer cells, apoptosis induction was assessed using the Annexin V-FITC kit according to the manufacturer's instructions. BxPC3 cells were exposed to DMSO, oridonin $(40 \mu \mathrm{M})$, or gemcitabine $(20 \mu \mathrm{M})$ either alone or in combination with oridonin, for $48 \mathrm{~h}$. Floating and adherent cells were collected by centrifugation at $1,000 \times \mathrm{g}$ for $5 \mathrm{~min}$. Pooled cells were washed with the manufacturer-supplied binding buffer. Approximately $5 \times 10^{4}$ cells were suspended in $0.2 \mathrm{~mL}$ of binding buffer containing Annexin V-FITC/PI. After 15 min of incubation in the dark, the fluorescence intensity of more than 10,000 viable cells from each sample was analyzed using a Coulter Epics XL flow cytometer with excitation and emission settings of 488 and $525 \mathrm{~nm}$, respectively. The data were analyzed using Cell quest software.

\section{Mitochondrial and cytosolic fractionation}

The mitochondrial and cytoplasmic fractions were separated using the mitochondrial/cytosol fractionation kit according to the manufacturer's instructions. Briefly, after cells were incubated and harvested, whole-cell pellets were dissolved in cytosolic fraction extraction buffer and subjected to 55 strokes in a $2 \mathrm{~mL}$ Dounce homogenizer on ice. The homogenate was centrifuged at 3,500 rpm for $10 \mathrm{~min}$ at $4{ }^{\circ} \mathrm{C}$ to pellet nuclei and unbroken cells. The supernatant was subsequently centrifuged at $13,000 \mathrm{rpm}$ for $30 \mathrm{~min}$ at $4{ }^{\circ} \mathrm{C}$ to obtain a cytosolic supernatant and a mitochondrial pellet. Mitochondrial pellets were resuspended in mitochondrial extraction buffer by gently vortexing for $30 \mathrm{~s}$.

\section{Western blotting}

Briefly, cells were incubated with oridonin $(40 \mu M)$ or gemcitabine $(20 \mu \mathrm{M})$, either alone or combination for $48 \mathrm{~h}$. Untreated cells were used as controls. Subsequently, cells were harvested, washed twice with ice-cold PBS, and the cell pellets were resuspended in a lysis buffer consisting of 50 mM HEPES (pH 7.4), 1\% (v/v) Triton-X 100, 2 mM sodium orthovanadate, $100 \mathrm{mM}$ sodium fluoride, $1 \mathrm{mM}$ edetic acid, $1 \mathrm{mM}$ PMSF, $10 \mathrm{mg} / \mathrm{L}$ aprotinin, and $10 \mathrm{mg} / \mathrm{L}$ leupeptin (all from Sigma) and lysed at $4{ }^{\circ} \mathrm{C}$ for $60 \mathrm{~min}$. Cells were centrifuged at $13,000 \mathrm{rpm}$ for $15 \mathrm{~min}$ and the protein content of the supernatant was determined using a bicinchoninic acid (BCA) assay kit (Sigma) according to the manufacturer's instructions. Protein lysates $(20 \mu \mathrm{g} / \mathrm{lane})$ were separated by electrophoresis on a $12 \%$ SDS polyacrylamide gel and blotted onto a nitrocellulose membrane. Each membrane was blocked with 5\% skimmed milk for $2 \mathrm{~h}$ and incubated with primary antibodies against Bax, Smac, XIAP, caspase- 9 , caspase- 3 , and $\beta$-actin overnight at $4{ }^{\circ} \mathrm{C}$. The membrane was then incubated with secondary antibodies, goat anti-rabbit or goat anti-mouse IgG conjugated with horseradish peroxidase (HRP) for $1 \mathrm{~h}$ at room temperature. The formed immunocomplex was visualized by an enhanced chemiluminescence detection system (Beyotime, Shanghai, China) and exposed to X-ray film.

\section{Experimental animals}

Female nude BALB/cA-nu (nu/nu) mice (4-6 weeks old; weight 16-18 g) were purchased from Shanghai Cancer Institute for Tumor Implantation and maintained in a specific-pathogen-free environment at the experimental animal center of Zhejiang Chinese Medical University. The temperature for the animals was controlled at $25 \pm 1{ }^{\circ} \mathrm{C}$ and 
the relative humidity controlled at $40-60 \%$. All procedures and handing of animals in this study were performed according to local guidelines for the care of laboratory animals of Animal Experimental Center and were approved by the ethics committee for research on laboratory animal use of the Zhejiang Chinese Medical University (ZSLL2016-91).

\section{Murine model establishment and experimental scheme}

BxPC-3 cells in log-phase were added to serum-free culture medium at a density of $5 \times 10^{6}$ cells in $0.2 \mathrm{~mL}$. Tumor xenografts were established by subcutaneous inoculation of BxPC-3 pancreatic cancer cells into the right abdominal flanks of 40 nude mice. Tumors were left to develop for 5 weeks until they reached a size of $100-150 \mathrm{~mm}^{3}$, at which point treatment was initiated. Mice were randomly divided into four treatment groups with 10 mice per group and treated with $0.2 \mathrm{~mL}$ saline, gemcitabine $(80 \mathrm{mg} / \mathrm{kg})$ alone, oridonin $(40 \mathrm{mg} / \mathrm{kg})$ alone, or gemcitabine $(80 \mathrm{mg} / \mathrm{kg})$ and oridonin $(40 \mathrm{mg} / \mathrm{kg})$ in combination every 3 days for up to 30 days via intraperitoneal injection. The body weight and tumor size of mice were measured every 6 days following treatment. Tumor volume was measured using calipers and estimated according to the formula: tumor volume $\left(\mathrm{mm}^{3}\right)=$ $\mathrm{L} \times \mathrm{W}^{2} / 2$, where $\mathrm{L}$ is the length, and $W$ is the width. One week after the final treatment, mice were sacrificed, and tumors were excised. Xenograft tumors were harvested and fixed in $4 \%$ formalin, embedded in paraffin, and cut into suitable sections for the immunohistochemistry, Western blot, and RT-PCR analysis.

\section{Immunobistochemistry assay}

Sections of paraffin-embedded tumor tissues were routinely deparaffinized and rehydrated prior to nonspecific antigen blocking with goat serum. Immunostaining was performed using primary antibodies specific for Bax, Smac, XIAP, caspase-9, cleaved caspase-3 with appropriate dilutions, followed by staining with the appropriate HRPconjugated secondary antibodies. Slides were developed in diaminobenzidine and counterstained with a weak solution of hematoxylin. Stained slides were dehydrated, mounted in Permount, and visualized on a microscope. At least 10 fields were randomly selected from each section. Images were captured with an attached camera linked to a computer and analyzed by Image-Pro Plus 6.0 software for data quantification.

\section{RT-PCR assay in tumor tissues}

Total RNA was extracted from tumor tissues using Trizol reagent. The amount and purity of extracted RNA was quantified by spectrophotometry. The value of A260/ A280 was measured to evaluate the quality of RNA. cDNA was synthesized with $5 \mu \mathrm{g}$ of total RNA and oligo(dT) primers according to the manufacturer's instructions. PCR amplification was performed using the GeneAmp PCR System 9600 (PERKIN-ELMER Corp. Norwalk, CT, USA).

PCR amplification conditions:

* Bax: $94^{\circ} \mathrm{C} 30 \mathrm{~s}, 57^{\circ} \mathrm{C} 30 \mathrm{~s}, 72^{\circ} \mathrm{C} 20 \mathrm{~s}, 30$ cycles;

* Smac: $94^{\circ} \mathrm{C} 30 \mathrm{~s}, 56^{\circ} \mathrm{C} 30 \mathrm{~s}, 72^{\circ} \mathrm{C} 30 \mathrm{~s}, 30$ cycles;

* XIAP: $94^{\circ} \mathrm{C} 30 \mathrm{~s}, 58^{\circ} \mathrm{C} 20 \mathrm{~s}, 72^{\circ} \mathrm{C} 20 \mathrm{~s}, 30$ cycles;

* Caspase-9: $94{ }^{\circ} \mathrm{C} 30 \mathrm{~s}, 56{ }^{\circ} \mathrm{C} 30 \mathrm{~s}, 72{ }^{\circ} \mathrm{C} 30 \mathrm{~s}$, 35 cycles;

* Caspase-3: $94{ }^{\circ} \mathrm{C} 30 \mathrm{~s}, 57^{\circ} \mathrm{C} 30 \mathrm{~s}, 72{ }^{\circ} \mathrm{C} 30 \mathrm{~s}$, 35 cycles;

* GAPDH: $94^{\circ} \mathrm{C} 30 \mathrm{~s}, 54{ }^{\circ} \mathrm{C} 30 \mathrm{~s}, 72{ }^{\circ} \mathrm{C} 20 \mathrm{~s}, 25$ cycles.

GAPDH was used as an internal control. The primers used for the amplification are listed in Table 1. PCR products $(5 \mu \mathrm{L})$ were analyzed by electrophoresis on $1 \%$ agarose gel with ultraviolet (UV) illumination and the results were imaged.

\section{Statistical analysis}

All results were validated in at least three independent experiments. The data are expressed as means \pm S.D. Statistical comparisons were made by one-way ANOVA and Student's t-test using SPSS v17.0 software. $\mathrm{P}<0.05$ was considered statistically significant.

\section{Results}

\section{Oridonin inbibited proliferation of BxPC-3 and Panc-1 cells}

The CCK-8 assay was performed to evaluate the effect of oridonin on the proliferation of the BxPC-3 and Panc- 1 cell line. Oridonin inhibited the growth of pancreatic cancer cells in a concentration- and time-dependent manner (Figure 1A). Treating BxPC-3 cells with $40 \mu \mathrm{M}$ oridonin for $48 \mathrm{~h}$ could inhibit proliferation by $50.4 \%$ (Figure $1 A$ ). Oridonin at this concentration was used for all subsequent experiments.

In addition, Panc- 1 cells were treated with different concentrations of oridonin for $48 \mathrm{~h}$, oridonin induced changes to the cellular morphology. The number of nonadherent and potentially apoptotic cells were observed to 
Table 1 Sequences of primers used in the real-time PCR

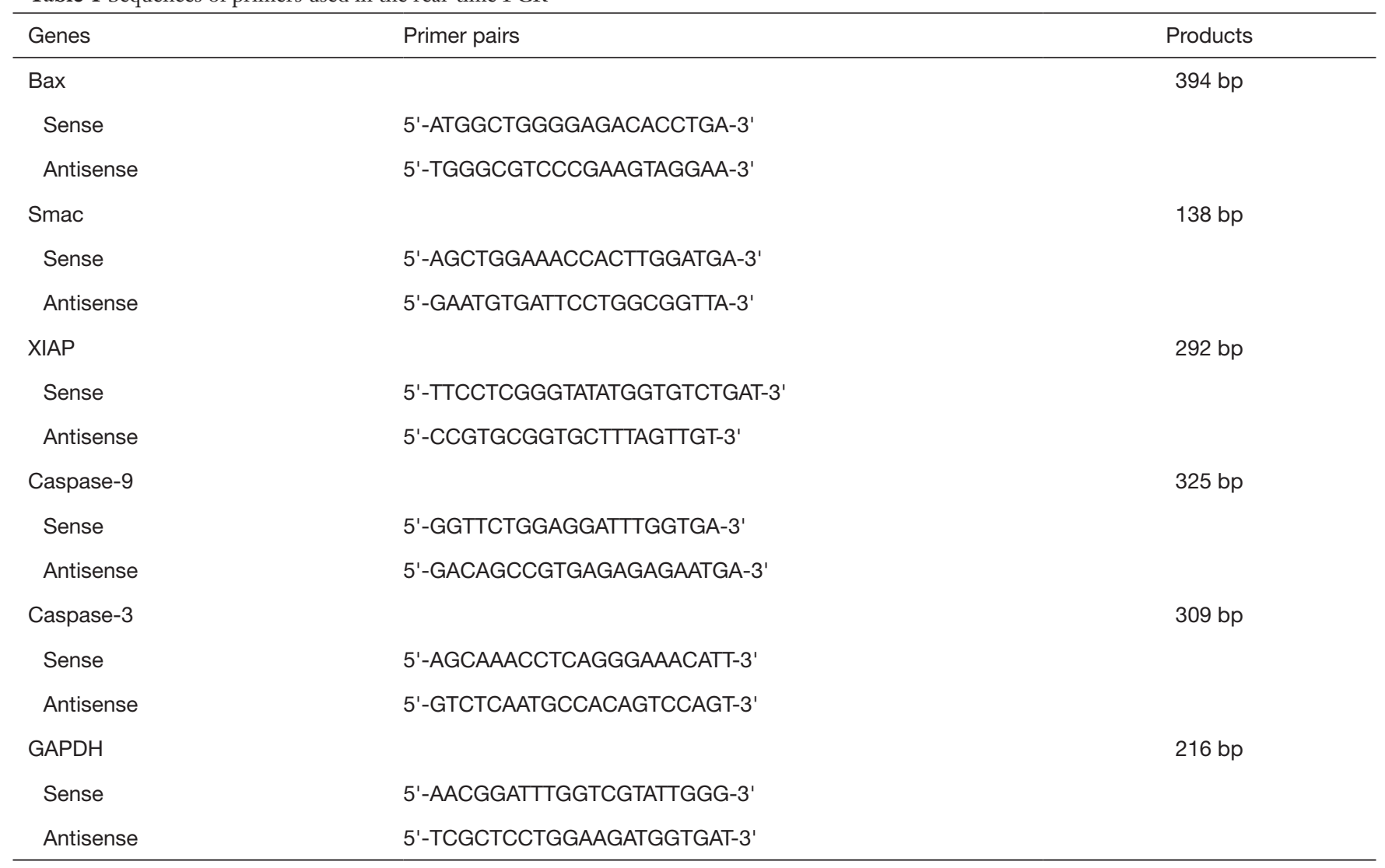

increase in comparison to adherent cells (Figure 1B).

\section{Oridonin inbibited proliferation and enbanced apoptosis of BxPC-3 in combination with gemcitabine}

To determine whether oridonin enhances inhibitory effects on proliferation and the induction of apoptosis by gemcitabine. CCK-8 and flow cytometric analysis was performed. BXPC-3 cells were treated with oridonin and gemcitabine, either alone or in combination for $48 \mathrm{~h}$. As shown in Figure 2, oridonin and gemcitabine alone had weak effect on cell activity, the effect is obviously enhanced after the combination of the two. oridonin and gemcitabine alone had low effect on apoptosis; in combination, a synergistic effect on the induction of apoptosis was observed.

\section{Oridonin and gemcitabine treatment modifies the mRNA expression of Bax, Smac, XIAP, and XIAP-regulated genes in BxPC-3 cells}

To investigate the expression of apoptosis-related genes,
RT-PCR was conducted to detect mRNA expression of Bax, Smac, XIAP, and XIAP-regulated genes in BxPC3 cells. Oridonin alone, gemcitabine alone, or the two in combination could up-regulate the expression of Bax (mitochondrial), Smac (cytosolic), caspase-9, and caspase-3 mRNA compared to control (Figure 3; all $\mathrm{P}<0.05$ ). These increases were amplified by co-treatment $(\mathrm{P}<0.01)$. XIAP expression in the gemcitabine group was also significantly up-regulated compared with control group, whilst expression was decreased in the oridonin group and significantly down-regulated in the combination therapy group (Figure 3; $\mathrm{P}<0.05$ ).

\section{Oridonin and gemcitabine co-treatment enhances a Smac-dependent apoptotic pathway}

Smac/DIABLO, a novel regulator of apoptosis, is released from mitochondria into the cytosol following mitochondrial membrane damage. The important function of Smac/ DIABLO is to neutralize the inhibitory effects of XIAP on caspase activation (17). Smac mainly prevents interaction 

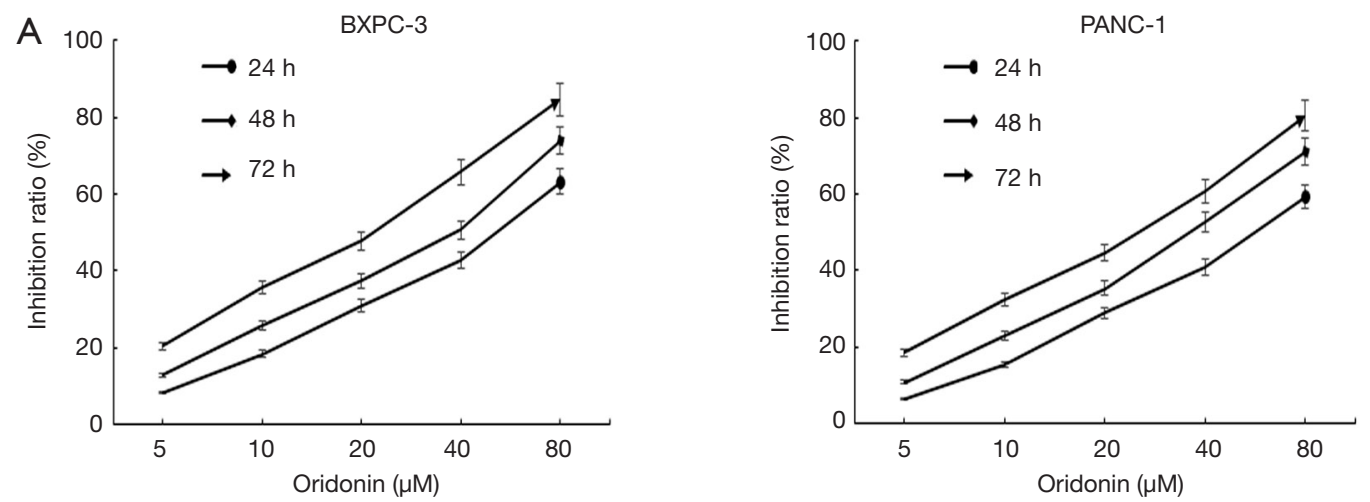

\section{B}
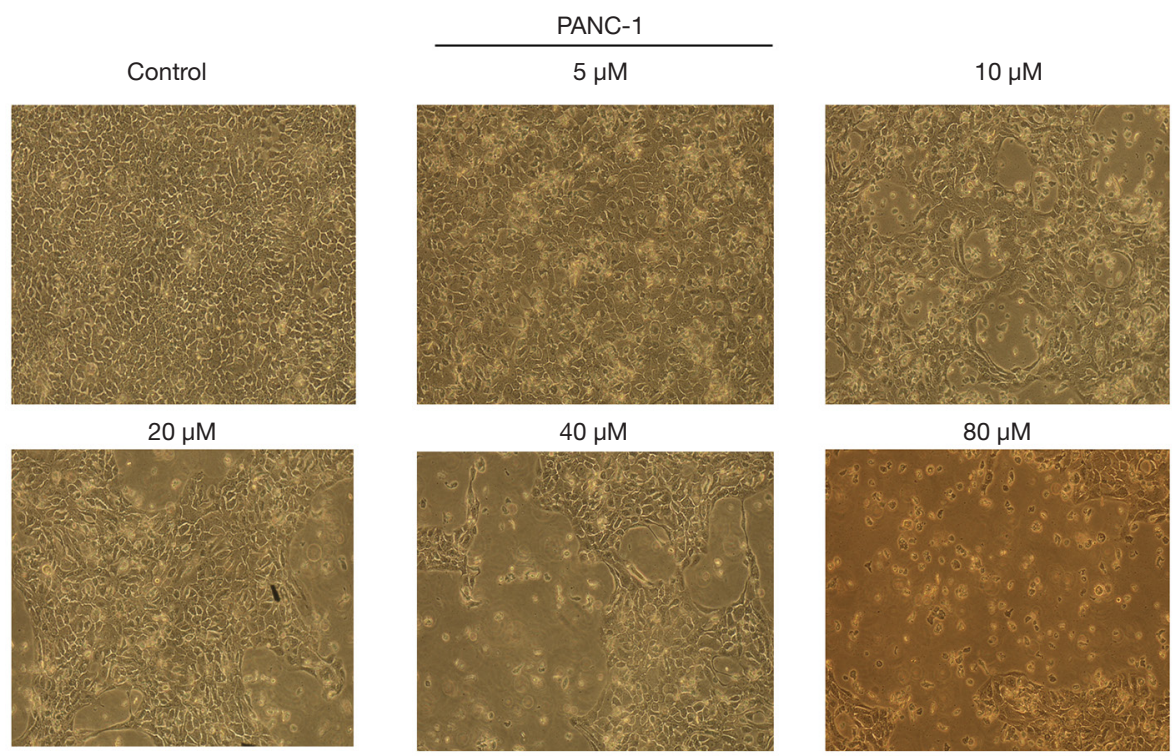

Figure 1 Effect of oridonin on inhibiting the growth of BxPC-3 and Panc-1 cells in vitro. (A) BxPC-3 and Panc-1 cells were treated with vehicle, 5, 10, 20, 40 or $80 \mu \mathrm{M}$ oridonin for $24 \mathrm{~h}, 48 \mathrm{~h}$, or $72 \mathrm{~h}$. Cell proliferation inhibition rates were determined by CCK-8 assay. (B) Panc-1 cells were treated with different concentrations oridonin for 48 h. cell morphology changes were observed under light microscope $(\times 200)$.

of XIAP with caspase-9 and subsequent activation of the apoptotic pathway (18). To elucidate the involvement of Smac and its related proteins in the induction of apoptosis following co-treatment with oridonin and gemcitabine, we conducted Western blot assays to first assess expression. Treatment with oridonin alone, gemcitabine alone, or in combination increased the protein expression of cytosolic Smac, caspase-9, and caspase-3 (all $\mathrm{P}<0.05$ ) compared to control cells (Figure 4). The combination of both was most effective. Interestingly, XIAP expression in the gemcitabine group was significantly upregulated compared with the control group. Conversely, XIAP expression was reduced in the oridonin group and down-regulated significantly in the combination therapy group (Figure 4; $\mathrm{P}<0.05$ ). These results suggested that the enhancement of gemcitabine-induced apoptosis by the addition of oridonin was predominantly achieved through activation of the Smac-dependent mitochondrial apoptotic pathway.

\section{Bax activation is necessary for apoptosis after gemcitabine and oridonin co-treatment}

Relocated Bax molecules facilitate mitochondrial release of Smac and cytochrome-c into the cytosol. To confirm whether Bax expression was linked to the anti-cancer effects of oridonin, Western blot analysis was conducted $48 \mathrm{~h}$ after the respective treatments. In untreated BxPC-3 cells, Bax was predominantly located in the cytosolic fraction (Figure 5). Oridonin or 
A

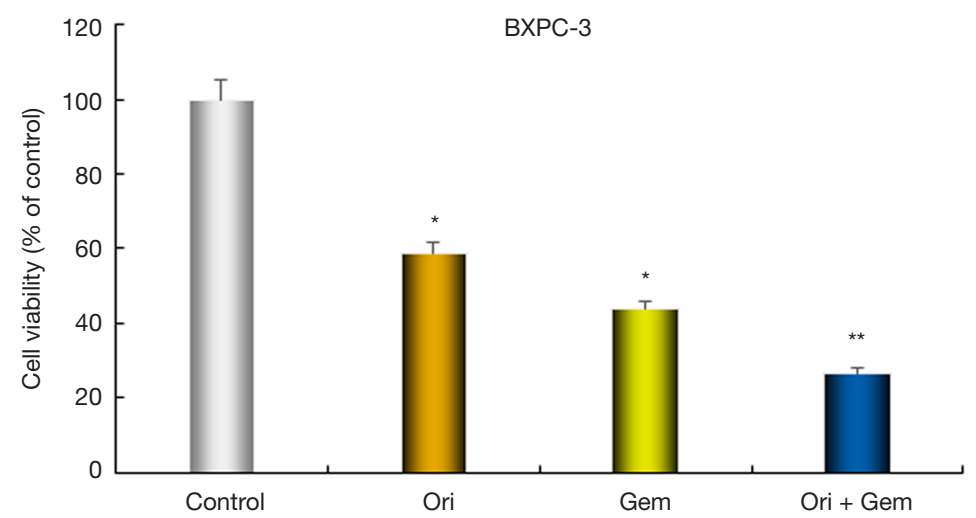

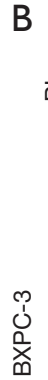

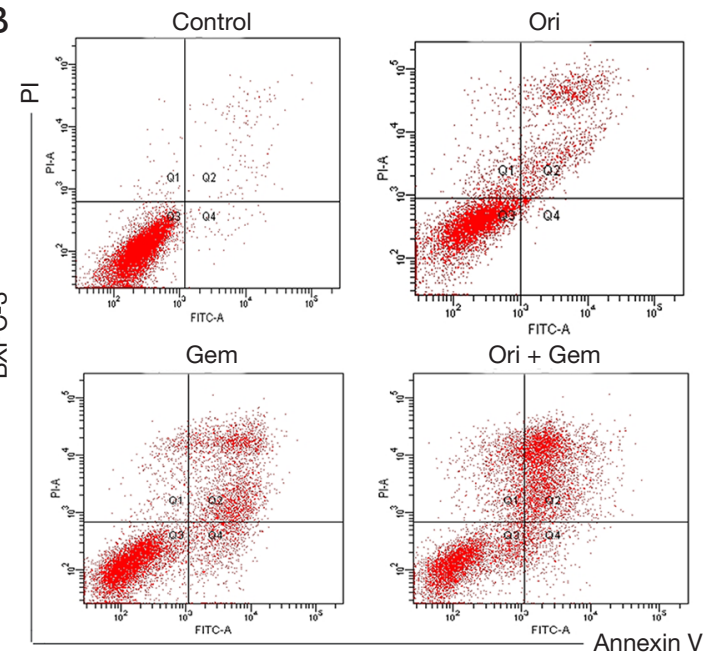

C

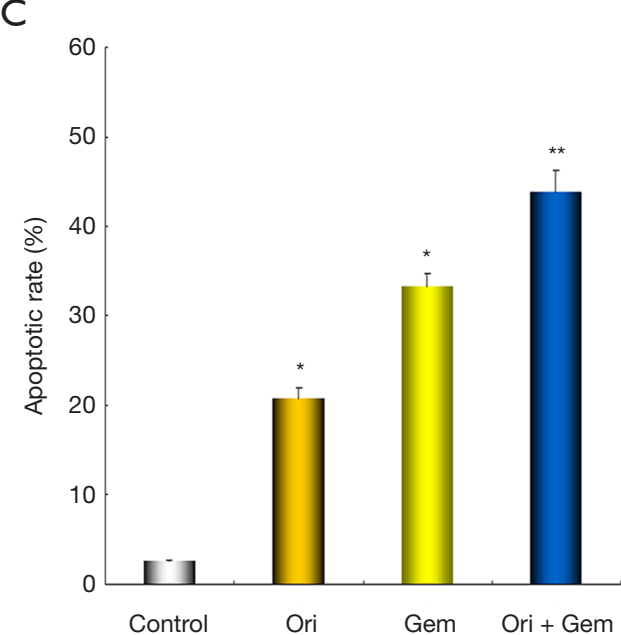

Figure 2 Effect of oridonin on cell viability and apoptosis of BxPC-3 cells in vitro. BxPC-3 cells were treated with oridonin $(40 \mu \mathrm{M})$ and gemcitabine $(20 \mu \mathrm{M})$ alone or combination for $48 \mathrm{~h}$. (A) Results of cell viability. (B and C) Results of flow cytometric analysis and the percentage of apoptotic cells is presented as the mean $\pm \mathrm{SD}$ of three independent experiments. ${ }^{*} \mathrm{P}<0.05$, ${ }^{* *} \mathrm{P}<0.01$ vs. the control or the cells treated with gemcitabine. Gem, gemcitabine; Ori, oridonin.

gemcitabine treatment alone induced Bax translocation from the cytosolic to the mitochondrial compartment (Figure 5). However, this change was more pronounced in the oridonin and gemcitabine combination group $(\mathrm{P}<0.05)$. The results indicated that Bax may participate in apoptosis induced by oridonin and gemcitabine in BxPC-3 cells.

\section{Oridonin and gemcitabine co-treatment reduced tumor volume and weight in nude mice}

Our in vitro data prompted us to examine whether the effects of oridonin and gemcitabine were demonstrable in vivo. We examined the effects of oridonin or gemcitabine alone, or in combination, on the growth of subcutaneously implanted tumors in nude mice. The experimental protocol is depicted in Figure 6. All nude mice survived for the duration of the treatment. Oridonin or gemcitabine treatment did not result in increased toxicity and no discernable effects on body weight were noted (Figure 6). Notably, tumor xenografts treated with oridonin, gemcitabine, or in combination were significantly decreased in both tumor volume and weight over the entire observation period compared to controls (Figure 6; $\mathrm{P}<0.05$ ).

\section{Bax, Smac, caspase-3, and caspase-9 were upregulated and XIAP down-regulated in tumor tissue following co-treatment}

To determine whether the impact on tumor growth 


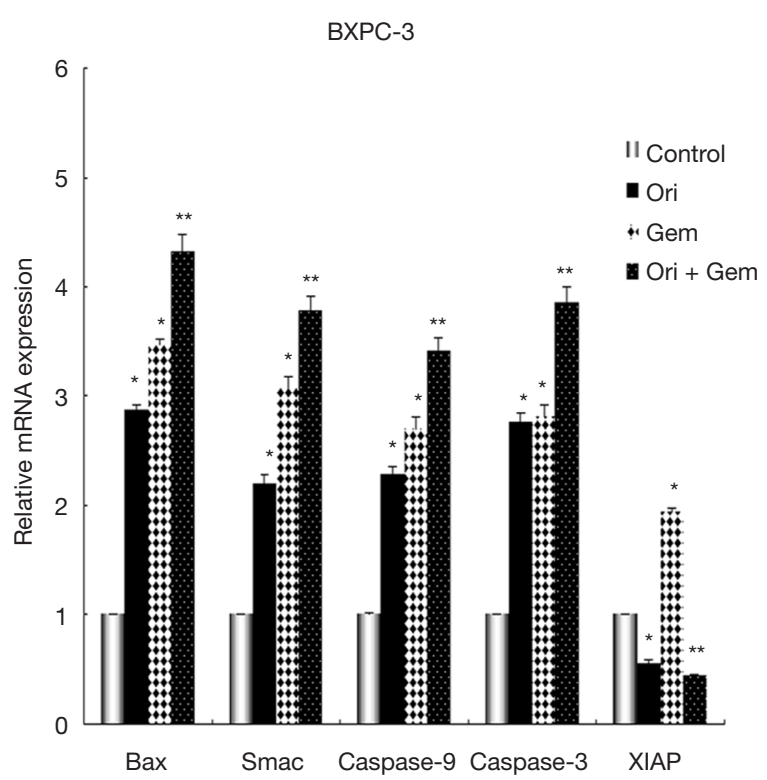

Figure 3 Effects of oridonin and gemcitabine on mRNA expression of Bax, Smac, XIAP, caspase-3, caspase-9 in pancreatic cancer cells in vitro. QPCR analysis demonstrated up-regulation of Bax, Smac, caspase-3, caspase-9 mRNA and the down-regulation of XIAP mRNA by either oridonin alone or in combination with gemcitabine. ${ }^{*} \mathrm{P}<0.05$ vs. control; ${ }^{*} \mathrm{P}<0.05$ vs. control or cells treated with gemcitabine alone. Gem, gemcitabine; Ori, oridonin. inhibition of oridonin was related to Bax, Smac, XIAP and XIAP-regulated proteins, we evaluated the effect of oridonin and gemcitabine on Bax, Smac, XIAP, caspase-9, caspase-3 expression by immunohistochemistry. Oridonin alone, or in combination with gemcitabine, significantly increased the expression of Bax, Smac, caspase- 9 and caspase-3 in tumor tissue compared controls (Figure 7; $\mathrm{P}<0.05)$. Conversely, expression of XIAP was up-regulated in the gemcitabine group and down-regulated in the oridonin and combination groups compared to that in control (Figure 7; $\mathrm{P}<0.05$ ). Importantly, the combination therapy group had the most pronounced effect $(\mathrm{P}<0.05)$ when compared with gemcitabine alone or control.

\section{Effects of oridonin and gemcitabine on the mRNA of Bax, Smac, XIAP and XIAP-regulated genes in vivo}

We investigated the mRNA expression of Bax, Smac, XIAP, and XIAP-regulated genes in tumor tissues. Both oridonin or gemcitabine alone significantly up-regulated the mRNA expression of Bax, Smac, caspase- 9 , caspase- 3 in tumor tissues compared to control (Figure $8 ; \mathrm{P}<0.05$ ). The combination therapy group induced the largest increase when compared with gemcitabine alone or control. XIAP
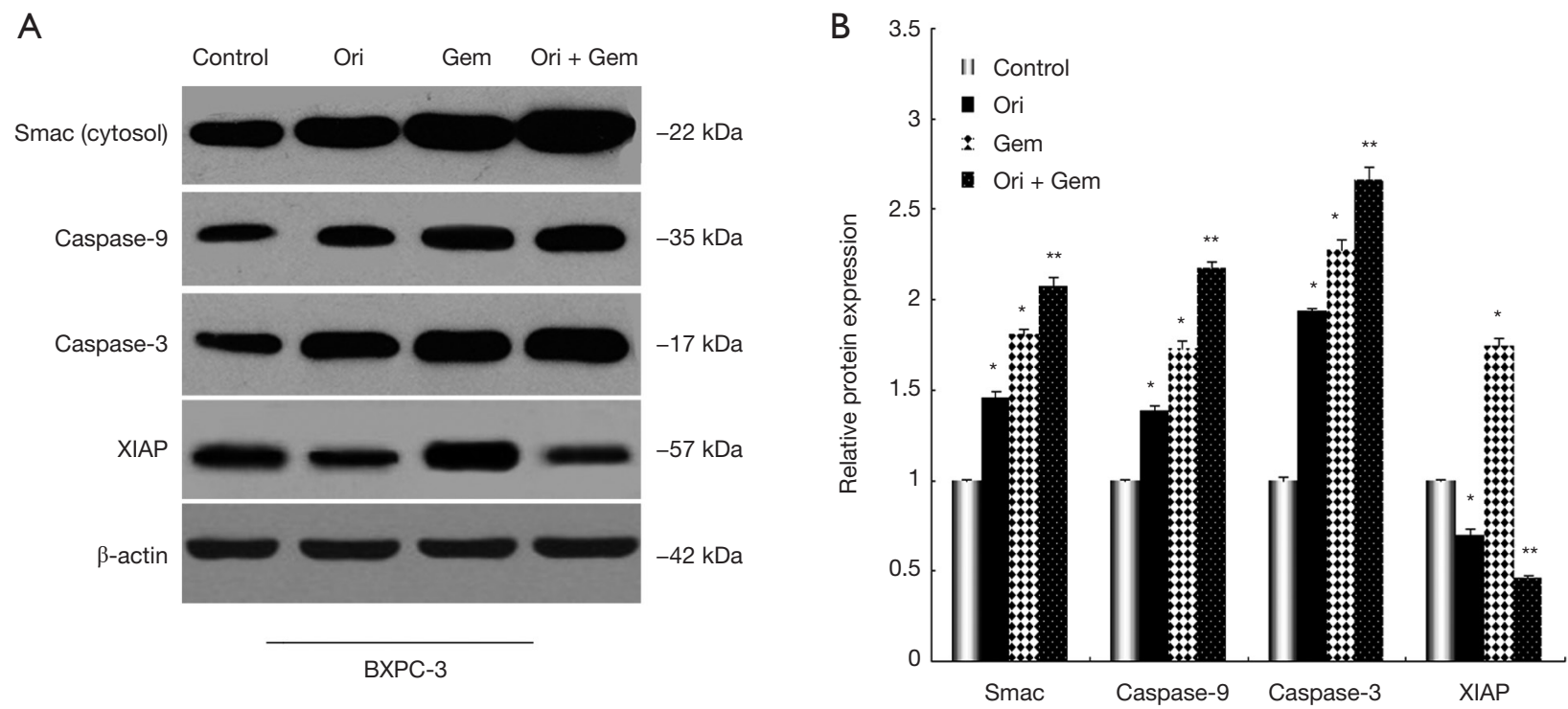

Figure 4 Oridonin potentiates the effect of gemcitabine in pancreatic cancer cells by activating Smac, which further promotes caspase activation by inhibiting XIAP expression in vitro. Cells were treated with oridonin alone or in combination with gemcitabine for 48 h. Smac, XIAP, caspase-3 and caspase-9 protein expressions were detected by Western blot analysis. (A) Results of western blot analysis; (B) quantified data from the Western blot. ${ }^{*} \mathrm{P}<0.05$ vs. controls. ${ }^{* *} \mathrm{P}<0.05$ vs. control or gemcitabine alone group. Gem, gemcitabine; Ori, oridonin. 

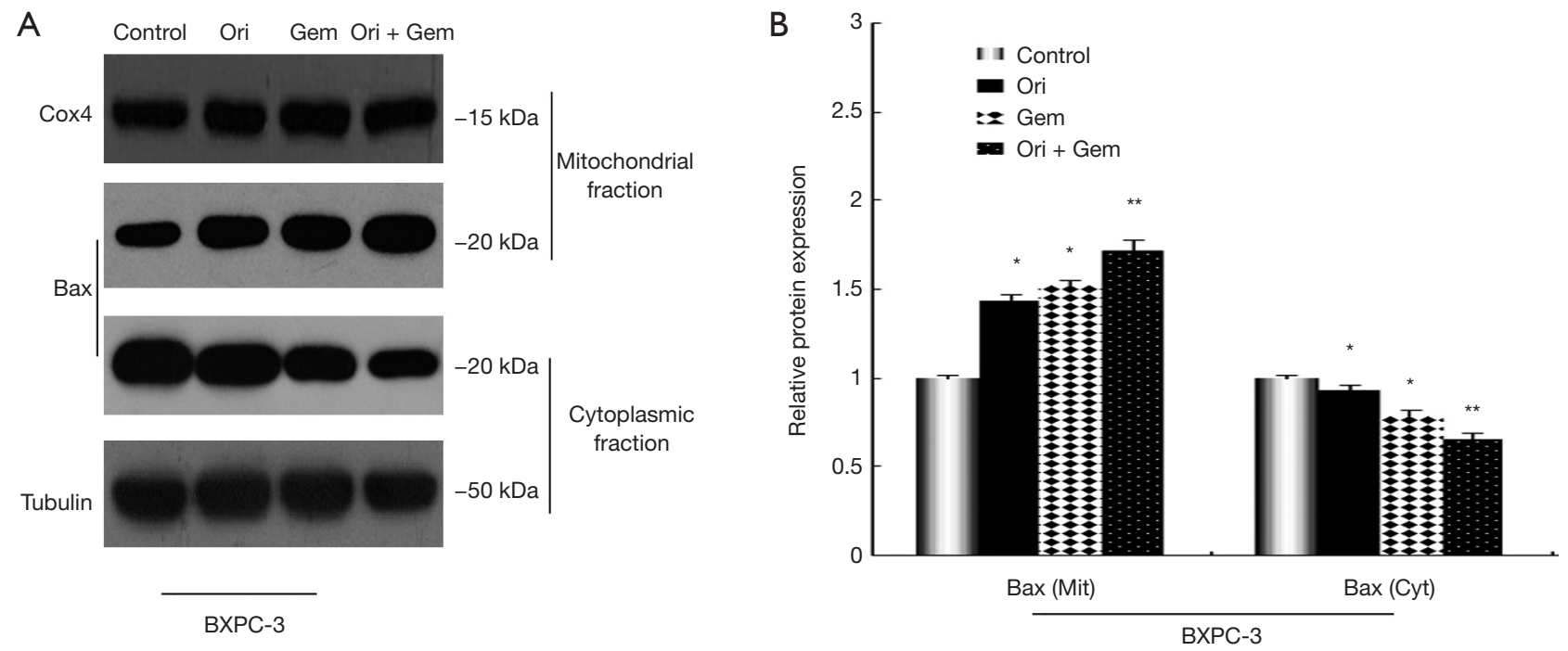

Figure 5 Bax plays an important role in oridonin- or gemcitabine-induced apoptosis. Cells were treated with oridonin alone or in combination with gemcitabine for 48 h, (A) Bax redistribution in BxPC-3 cells following treatment. Cox4 and Tubulin served as loading control for the mitochondrial and cytoplasmic fractions, respectively. Western blots are representative of three independent experiments. (B) Quantified data from the western blot. ${ }^{*} \mathrm{P}<0.05 v s$. control. ${ }^{* *} \mathrm{P}<0.05 v s$. control or gemcitabine alone group. Gem, gemcitabine; Ori, oridonin.

expression in the gemcitabine group was significantly upregulated but significantly decreased in the oridonin and combination therapy groups compared to controls (Figure 8; $\mathrm{P}<0.05)$.

\section{Discussion}

Although gemcitabine is a first-line treatment for pancreatic cancer, emerging intrinsic resistance and acquired resistance to gemcitabine is critically hampering the efficacy of this cornerstone of pancreatic cancer chemotherapy (19). The present study identified that oridonin could work synergistically with gemcitabine to significantly enhance apoptosis of $\mathrm{BxPC}-3$ cell in vitro and reduce tumor size in a xenograft nude mouse model. This was demonstrated to be linked to increased expression or release of the proapoptotic proteins Bax, Smac, caspase-3, and caspase-9, combined with decreased expression of XIAP.

Oridonin has been demonstrated to increase tumor sensitivity to apoptosis-inducing drugs, including imatinib, arsenic trioxide, and gemcitabine (20-22). Studies have shown that Smac/DIABLO protein release can occur independently of cytochrome-c release from the mitochondria during apoptosis $(17,23)$. In our experiment, CCK-8 kit assay and flow cytometry analysis demonstrated that oridonin enhanced the antitumor effects of gemcitabine by induction of apoptosis. These results are consistent with those from previous reports showing the suppressive effects of oridonin alone or in combination with gemcitabine against pancreatic cancer proliferation $(16,22)$.

The primary regulators of the mitochondrial pathway of apoptosis are the pro- and anti-apoptotic members of the B-cell CLL/lymphoma-2 (Bcl-2) protein family, which interact at the mitochondrial outer membrane (MOM) and arbitrate a life or death (permeabilization) decision there (24). One of the key proteins in this process is the pro-apoptotic Bax protein localized in the cytosol, which upon stress-induced activation, translocates to the MOM where it can neutralize anti-apoptotic proteins such as the integral membrane Bcl-2 protein. After complete Bcl-2 neutralization, Bax molecules combine to form pores that enable the release of apoptogenic factors such as cytochrome c, Smac/DIABLO, and apoptosis inducing factor (AIF) into the cytosol to induce cell death (25). Many studies suggest that in the absence of Bax no cytosolic Smac release occurs (26), however there are reports that show that apoptosis inducers such as AT-101 directly target the mitochondria and trigger Smac release irrespective of Bax activation (27). In the present study, we observed an upregulation of Bax expression in the mitochondrial fraction 
A

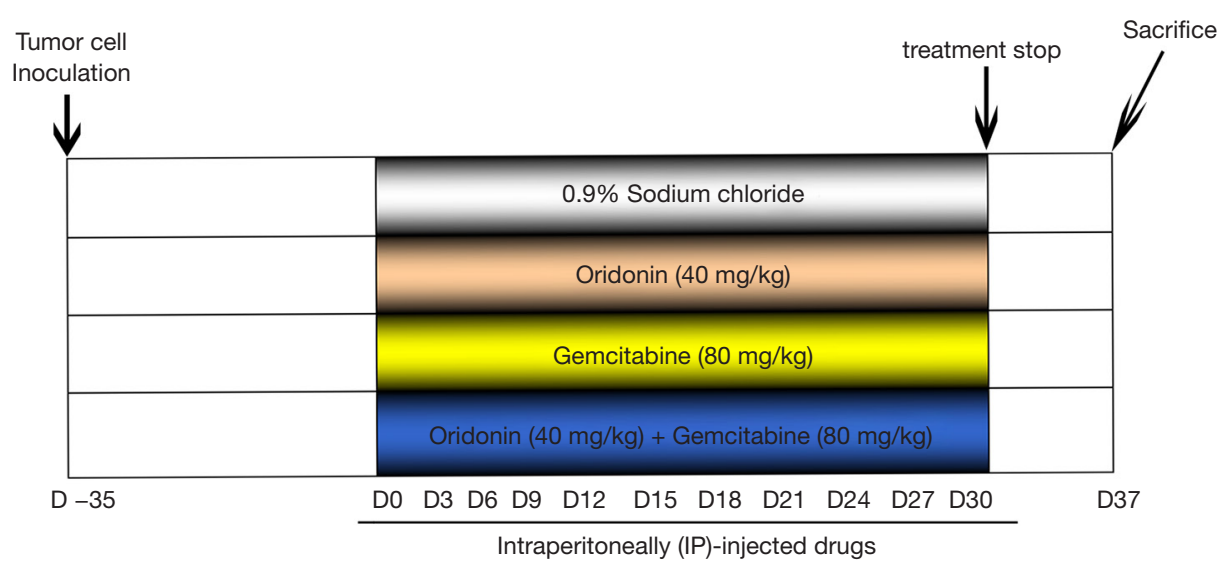

B
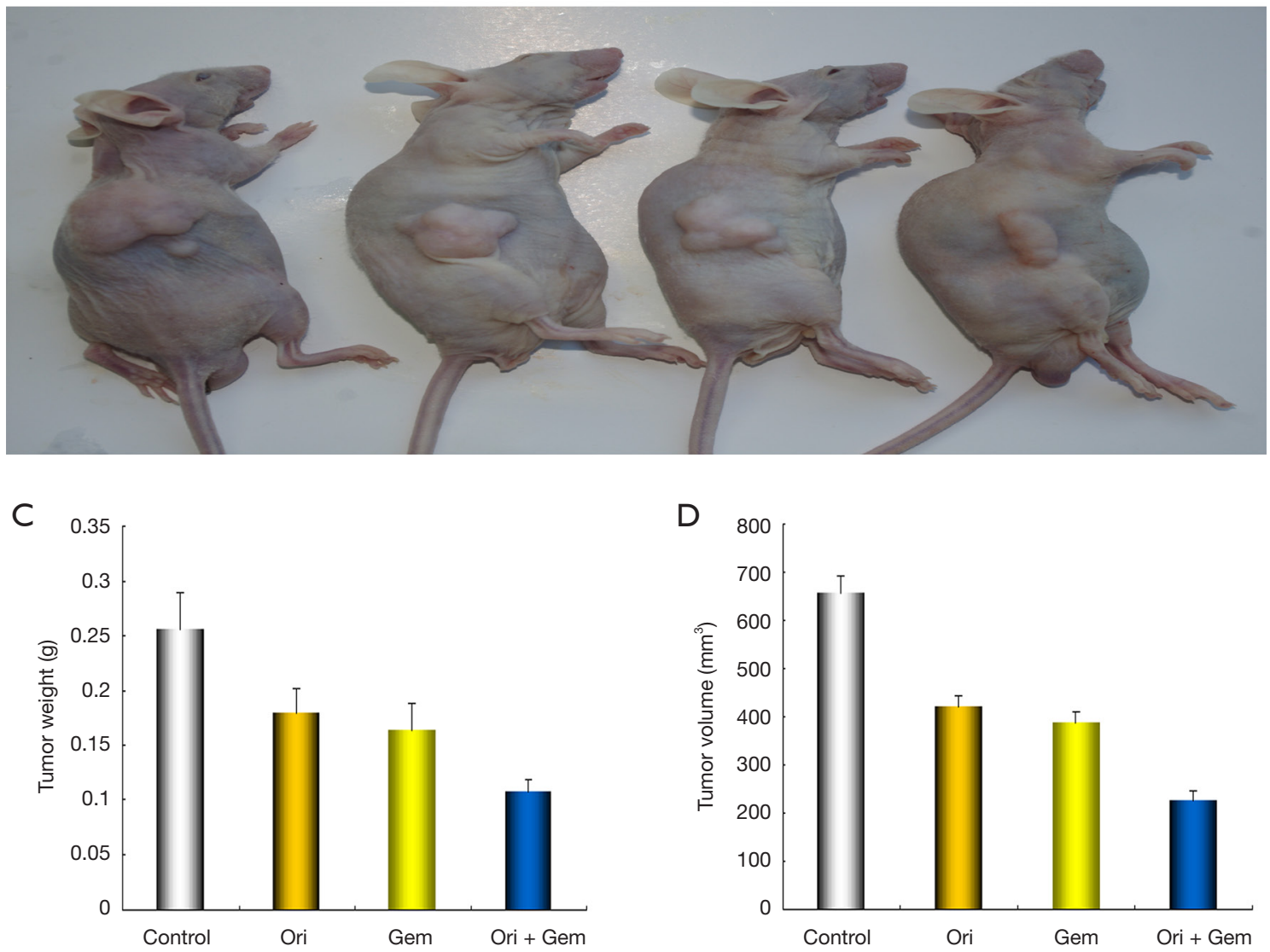

Figure 6 Oridonin potentiates the effect of gemcitabine to inhibit the growth of BXPC-3 xenograft in nude mice. (A) The experimental protocol described in Methods. (B) Photographs of mice bearing subcutaneously implanted pancreatic tumors subsequent to treatment with oridonin and gemcitabine. Compared with the control, treatment with oridonin, gemcitabine and a combination of the two demonstrated significant inhibition of tumor growth (C and D). One week after the last treatment, tumors were excised from the animals, and tumor volume were measured using calipers. The tumor weights and volumes of individual groups of mice are presented with a histogram. The data are representative of 3 independent experiments and expressed as the mean $\pm \mathrm{SD}$. ${ }^{*} \mathrm{P}<0.05 v$ s. control; ${ }^{* *} \mathrm{P}<0.05$ vs. control or the group treated with a single agent. D, day; Gem, gemcitabine; Ori, oridonin. 


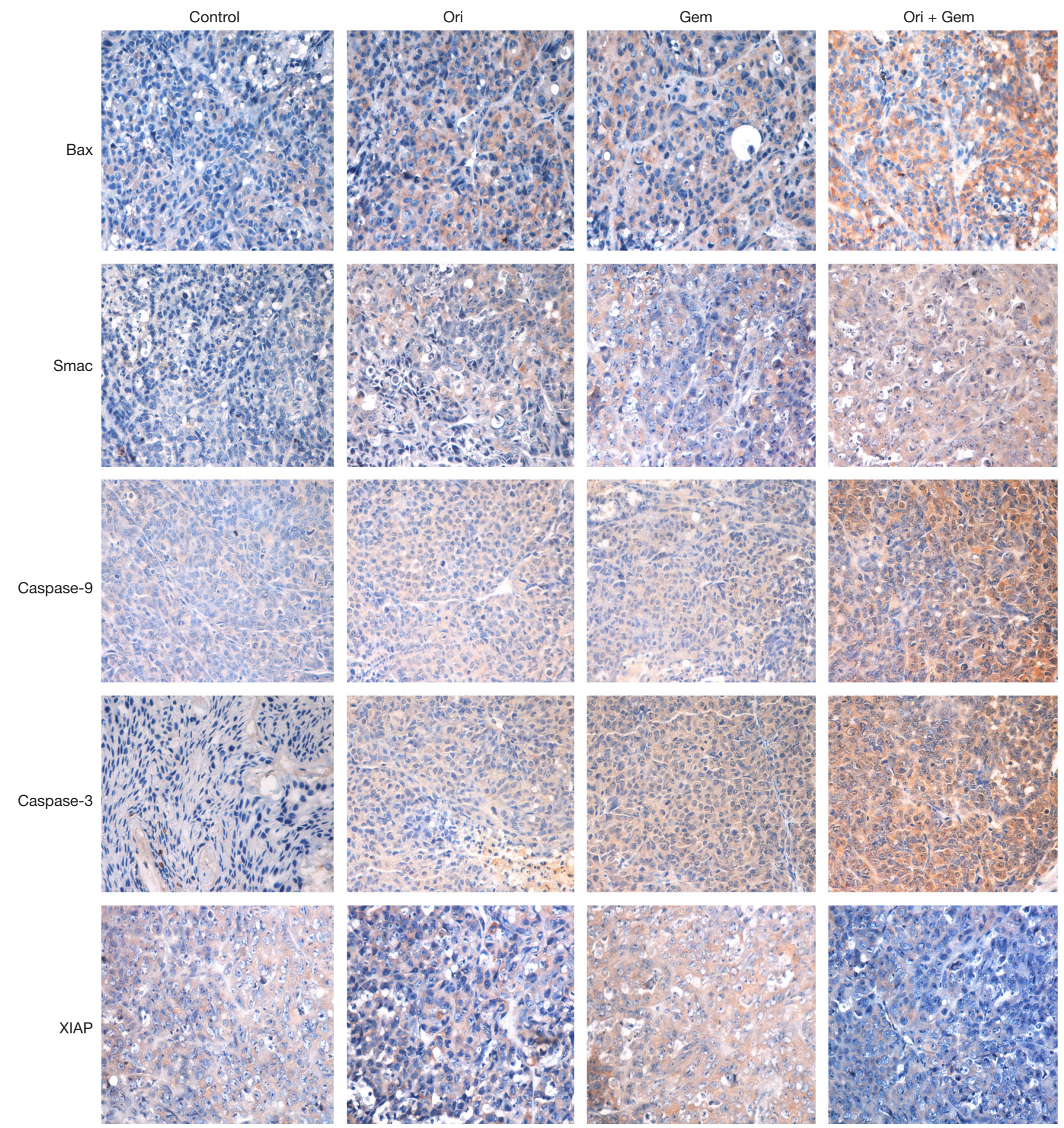

Figure 7 One week following final treatments, protein expression of Bax, Smac, XIAP, caspase-9 and caspase-3 were assessed using immunohistochemistry (magnification $\times 400$ ). Bax, Smac, caspase-9 and caspase-3 expression were up-regulated in tumor tissues treated with oridonin plus gemcitabine when compared with the control and gemcitabine-treated group $\left({ }^{*} \mathrm{P}<0.05\right)$. XIAP expressions were downregulated significantly in tumor tissues treated with oridonin plus gemcitabine when compared with the other three groups $\left({ }^{* *} \mathrm{P}<0.05\right)$. Gem, gemcitabine; Ori, oridonin. 

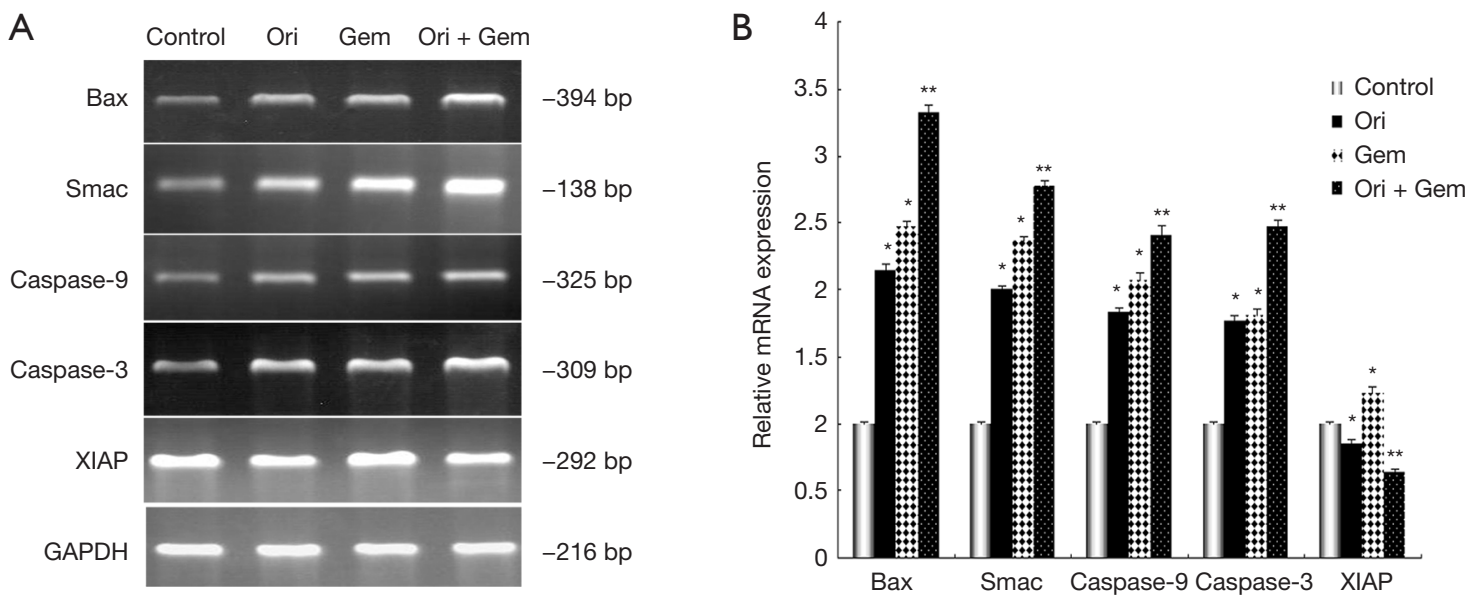

Figure $8 \mathrm{mRNA}$ levels of Bax, Smac, XIAP, and XIAP-regulated genes in tumor tissue in the different treatment groups were detected by RT-PCR. (A) Results of PCR analysis in tumor tissues; (B) quantified data from the PCR. ${ }^{*} \mathrm{P}<0.05$ vs. control; ${ }^{* *} \mathrm{P}<0.05$ vs. control or the mice treated with a single agent. Gem, gemcitabine; Ori, oridonin.

in oridonin and gemcitabine-treated BXPC-3 cells, which indicated that Bax might be a critical inducer of apoptosis following combined treatment. However, the relationship between Smac release and Bax activation is complex and is still under active investigation.

XIAP is the most potent caspase inhibitor of the IAP family, whose inhibitory effects in the mitochondria during apoptosis are well documented. In addition to its well-known function in caspase suppression, XIAP can permeabilize and enter mitochondria (28). High expression of this protein corresponds to poor prognosis and is correlated with disease progression in many cancers (29-31). XIAP was also reported to contribute to chemotherapy resistance, and targeting this protein was found to effectively sensitize cells to apoptosis and suppress tumor progression $(32,33)$. We identified that the expression levels of cytosolic Smac, caspase-9, and caspase- 3 were enhanced by cotreatment and this effect was accompanied by the downregulation of XIAP expression. Smac is a potent inhibitor of XIAP, which relieves caspase inhibition and allows apoptosis to proceed. Therefore, the enhanced expression of Smac in the cytosol, inhibition of XIAP, and the cleavage of proapoptotic caspases following the treatment of cells with oridonin and gemcitabine, is a likely mechanism underlying the synergistic effects observed.

In summary, our present findings indicate that oridonin potentiates the antitumor effects of gemcitabine by inducing mitochondrial release of the apoptogenic protein Smac in a Bax-dependent manner. Subsequently, this enhances inhibition of XIAP, cleavage of pro-apoptotic caspases, and enhanced apoptosis. Some molecular links require clarification, such as how Smac release is connected to Bax activation. To conclude, the combination of oridonin with gemcitabine has significant potential as an effective therapy for pancreatic cancer. Based on these results, further clinical studies are necessary to confirm our findings in patients with pancreatic cancer.

\section{Acknowledgments}

We thank the entire staff of the Animal Experimental Center in Zhejiang Chinese Medical University and scientific research platform of Zhejiang Chinese Medical University for helpful assistance.

Funding: The present study was supported by the Basic Public Welfare Research Project of Zhejiang (grant no. LGD19H160006); The Medical Scientific Technology Protect of Zhejiang (grant no.2018KY614); The National Natural Science Foundation of China (grant no. 81202821).

\section{Footnote}

Reporting Checklist: The authors have completed the ARRIVE reporting checklist. Available at http://dx.doi. org/10.21037/tcr-19-3000

Data Sharing Statement: Available at http://dx.doi. org/10.21037/tcr-19-3000

Conflicts of Interest: All authors have completed the ICMJE 
uniform disclosure form (available at http://dx.doi. org/10.21037/tcr-19-3000). The authors have no conflicts of interest to declare.

Ethical Statement: The authors are accountable for all aspects of the work in ensuring that questions related to the accuracy or integrity of any part of the work are appropriately investigated and resolved. All procedures and handing of animals in this study were performed according to local guidelines for the care of laboratory animals of Animal Experimental Center and were approved by the ethics committee for research on laboratory animal use of the Zhejiang Chinese Medical University (ZSLL-2016-91).

Open Access Statement: This is an Open Access article distributed in accordance with the Creative Commons Attribution-NonCommercial-NoDerivs 4.0 International License (CC BY-NC-ND 4.0), which permits the noncommercial replication and distribution of the article with the strict proviso that no changes or edits are made and the original work is properly cited (including links to both the formal publication through the relevant DOI and the license). See: https://creativecommons.org/licenses/by-nc-nd/4.0/.

\section{References}

1. Siegel RL, Miller KD, Jemal A. cancer statistics 2017. CA Cancer J Clin 2017;67:7-30.

2. Lam ET, Bracci PM, Holly EA, et al. Mitochondrial DNA sequence variation and risk of pancreatic cancer. Cancer Res 2012;72:686-95.

3. Norsa' adah B, Nur-Zafira A, Knight A. Pancreatic cancer in Universiti Sains Malaysia Hospital: a retrospective review of years 2001-2008. Asian Pac J Cancer Prev 2012;13:2857-60.

4. Werner J, Büchler MW. Management of pancreatic cancer: recent advances. Dtsch Med Wochenschr 2011;136:1807-10.

5. Warsame R, Grothey A. Treatment options for advanced pancreatic cancer: a review. Expert Rev Anticancer Ther 2012;12:1327-36.

6. Conroy T, Desseigne F, Ychou M, et al. Folfirinox versus gemcitabine for metastatic pancreatic cancer. $\mathrm{N}$ Engl J Med 2011;364:1817-25.

7. Tholey R, Sawicki JA, Brody JR. Molecular-based and alternative therapies for pancreatic cancer: looking "out of the box". Cancer J 2012;18:665-73.

8. Tan W, Lu J, Huang M, et al. Anti-cancer natural products isolated from chinese medicinal herbs. Chin Med 2011;6:27.

9. Wang S, Zhong Z, Wan J, et al. Oridonin induces apoptosis, inhibits migration and invasion on highlymetastatic human breast cancer cells. Am J Chin Med 2013;41:177-96.

10. Chen G, Wang K, Yang BY, et al. Synergistic antitumor activity of oridonin and arsenic trioxide on hepatocellular carcinoma cells. Int J Oncol 2012;40:139-47.

11. Bao R, Shu $\mathrm{Y}, \mathrm{Wu} X$, et al. Oridonin induces apoptosis and cell cycle arrest of gallbladder cancer cells via the mitochondrial pathway. BMC Cancer 2014;14:217.

12. Sun KW, Ma YY, Guan TP, et al. Oridonin induces apoptosis in gastric cancer through Apaf-1, cytochrome c and caspase- 3 signaling pathway. World J Gastroenterol 2012;18:7166-74.

13. Bu HQ, Liu DL, Wei WT, et al. Oridonin induces apoptosis in SW1990 pancreatic cancer cells via p53- and caspase-dependent induction of p38 MAPK. Oncol Rep 2014;31:975-82.

14. Muñoz-Pinedo C. Signaling pathways that regulate life and cell death: evolution of apoptosis in the context of selfdefense. Adv Exp Med Biol 2012;738:124-43.

15. Xiong S, Mu T, Wang G, et al. Mitochondria-mediated apoptosis in mammals. Protein Cell 2014;5:737-49.

16. Liu DL, Bu HQ, Jin HM, et al. Enhancement of the effects of gemcitabine against pancreatic cancer by oridonin via the mitochondrial caspase-dependent signaling pathway. Mol Med Rep 2014;10:3027-34.

17. Goyal S, Amar SK, Dwivedi A, et al. Photosensitized 2-amino-3- hydroxypyridine-induced mitochondrial apoptosis via Smac/DIABLO in human skin cells. Toxicol Appl Pharmacol 2016;297:12-21.

18. Obexer P, Ausserlechner MJ. X-linked inhibitor of apoptosis protein-a critical death resistance regulator and therapeutic target for personalized cancer therapy. Front Oncol 2014;4:197.

19. Grasso C, Jansen G, Giovannetti E. Drug resistance in pancreatic cancer: Impact of altered energy metabolism. Crit Rev Oncol Hematol 2017;114:139-52.

20. Guo Y, Shan Q, Gong Y, et al. Oridonin in combination with imatinib exerts synergetic anti-leukemia effect in $\mathrm{Ph}+$ acute lymphoblastic leukemia cells in vitro by inhibiting activation of LYN-mTOR signaling pathway. Cancer Biol Ther 2012;13:1244-54.

21. Chen G, Wang K, Yang BY, et al. Synergistic antitumor activity of oridonin and arsenic trioxide on hepatocellular carcinoma cells. Int J Oncol 2012;40:139-47. 
22. Bu HQ, Luo J, Chen H, et al. Oridonin enhances antitumor activity of gemcitabine in pancreatic cancer through MAPK-p38 signaling pathway. Int J Oncol 2012;41:949-958.

23. Li T, Zeng L, Gao W, et al. PSAP induces a unique Apaf1 and Smac-dependent mitochondrial apoptotic pathway independent of Bcl-2 family proteins. Biochim Biophys Acta 2013;1832:453-74.

24. Martinou JC, Youle RJ. Mitochondria in apoptosis: Bcl2 family members and mitochondrial dynamics. Dev Cell 2011;21:92-101.

25. Westphal D, Dewson G, Menard M, et al. Apoptotic pore formation is associated with in-plane insertion of Bak or Bax central helices into the mitochondrial outer membrane. Proc Natl Acad Sci U S A 2014;111:E4076-85.

26. Berens HM, Tyler KL. The proapoptotic Bcl-2 protein Bax plays an important role in the pathogenesis of reovirus encephalitis. J Virol 2011;85:3858-71.

27. Hu W, Wang F, Tang J, et al. Proapoptotic protein Smac mediates apoptosis in cisplatin-resistant ovarian cancer cells when treated with the anti-tumor agent AT101. J Biol Chem 2012;287:68-80.

28. Hamacher-Brady A, Brady NR. Bax/Bak-dependent, Drp1independent Targeting of X-linked inhibitor of apoptosis

Cite this article as: Liu DL, Bu HQ, Wang WL, Luo H, Cheng BN. Oridonin enhances the anti-tumor activity of gemcitabine towards pancreatic cancer by stimulating Bax- and Smac-dependent apoptosis. Transl Cancer Res 2020;9(7):41484161. doi: 10.21037/tcr-19-3000 protein XIAP) into inner mitochondrial compartments counteracts smac/DIABLO-dependent effector caspase activation. J Biol Chem 2015;290:22005-18.

29. Yang XH, Feng ZE, Yan M, et al. XIAP Is a Predictor of Cisplatin-Based Chemotherapy Response and Prognosis for Patients with Advanced Head and Neck Cancer. PLoS One 2012;7:e31601.

30. Hundsdoerfer P, Dietrich I, Schmelz K, et al. XIAP expression is post-transcriptionally upregulated in childhood ALL and is associated with glucocorticoid response in T-cell ALL. Pediatr Blood Cancer 2010;55:260-6.

31. Zhou S, Ye W, Shao Q, et al. Prognostic significance of $\mathrm{XIAP}$ and $\mathrm{NF}-\kappa \mathrm{B}$ expression in esophageal carcinoma with postoperative radiotherapy. World J Surg Oncol 2013;11:288.

32. Paschall AV, Zimmerman MA, Torres CM, et al. Ceramide targets xIAP and cIAP1 to sensitize metastatic colon and breast cancer cells to apoptosis induction to suppress tumor progression. BMC Cancer 2014;14:24.

33. Miyamoto M, Takano M, Iwaya K, et al. X-chromosomelinked inhibitor of apoptosis as a key factor for chemoresistance in clear cell carcinoma of the ovary. Br J Cancer 2014;110:2881-6. 